\title{
The Role of Complement System in Graft versus Host Disease
}

Mohamad A Cherry ${ }^{1}$, Hiral Parekh ${ }^{1}$, Megan Lerner ${ }^{2}$, Zhongxin Yu ${ }^{3}$, Sarah K Vesely ${ }^{4}$, George Selby ${ }^{1}$ and Jennifer Holter ${ }^{1,5^{*}}$

${ }^{1}$ Division of Hematology and Oncology, University Health Sciences Center (OUHSC), Oklahoma City, OK, USA

${ }^{2}$ Department of Surgery, University Health Sciences Center (OUHSC), Oklahoma City, OK, USA

${ }^{3}$ Department of Pathology, University Health Sciences Center (OUHSC), Oklahoma City, OK, USA

${ }^{4}$ Department of Biostatistics and Epidemiology, College of Public Health Oklahoma, USA

${ }^{5}$ Department of Radiation Oncology, University Health Sciences Center (OUHSC), Oklahoma City, OK, USA

"Corresponding author: Jennifer Holter, MD, The Oklahoma University Stephenson Cancer Center, Oklahoma City, OK 73104, USA, Tel: 405-271-4022; Fax: 405-271-4221; E-mail: Jennifer-Holter-Chakrabarty@ouhsc.edu

Received date: Mar 31, 2015, Accepted date: Apr 25, 2015, Publication date: Apr 30, 2015

Copyright: (C) 2015 Cherry MA, et al. This is an open-access article distributed under the terms of the Creative Commons Attribution License, which permits unrestricted use, distribution, and reproduction in any medium, provided the original author and source are credited.

\begin{abstract}
The role of complement system in stem cell transplantation is largely unknown. In solid organ transplantation, endovascular C4d deposition, a degradation product of the classic complement pathway, is essential for early rejection diagnosis. We retrospectively analyzed all patients diagnosed with graft versus host disease (GVHD) for C4d deposition at Oklahoma University between years 2000 and 2008. A modified Banff07 grading system was used to quantify C4d deposition. 58 biopsies ( 40 skin, 18 colon) performed on patients clinically suspected of having GVHD and 12 controls (all colon biopsies) were analyzed for C4d deposition. We recorded "response to steroids" in all clinical GVHD cases and looked at whether C4d can be utilized as a predictor of steroid treatment response. Of 40 clinical skin GVHD cases, 27 showed positive C4d staining which did not correlate well with steroid sensitivity: $74 \%$ of positive C4d cases responded to steroid therapy compared to $92 \%$ for negative cases $(p=0.2634) .94 \%$ of colon GVHD cases showed positive C4d staining compared to $17 \%$ in controls $(p<0.001)$. Only $44 \%$ of clinical colon GVHD cases were confirmed pathologically by H\&E, compared to $93 \%$ of skin GVHD cases. For colon GVHD cases, $61 \%$ had clinical grade III/IV, and $78 \%$ responded to steroids. Interestingly, $90 \%$ of negative H\&E colon cases responded to steroids. In conclusion, C4d deposition is a valuable marker for detection of colon GVHD, indicating a potential role of complement system in the pathogenesis of GVHD. C4d staining is potentially an objective tool than can help pathologist, in addition to H\&E, to diagnosed colon GVHD.
\end{abstract}

Keywords: C4d deposition; Colon; GVHD; Pathogenesis; Steroid therapy

\section{Introduction}

Successful transplantation, both in solid organs and bone marrow, is reliant on a delicate balance between the immune system and "foreign body." In solid organ transplantation, the foreign body is the transplanted organ, whereas in bone marrow transplantation, the donor stem cells constitute the new immune system while the entire recipient is considered a foreign body. Acute and chronic rejection can occur in both systems. The rejections in both scenarios have been defined and diagnosed by the elements of the immune system that react, and the time course in which this reaction occurs. While established rejection has been defined by the $\mathrm{T}$ and $\mathrm{B}$ lymphocyte components of the immune system in both, the impact of complement system in transplant rejection resides almost completely in the literature of solid organ transplantation [1].

In solid organ transplantation, the elements of acute rejection have been identified and are commonly related to different key players of the immune system. In acute rejection, loss of graft is heavily dependent on the presence of pre-formed antibodies directed against the human leukocyte antigen system (HLA). The pathologic diagnosis of acute rejection is defined by the presence of these antibodies and depends on the demonstration of $\mathrm{C} 4 \mathrm{~d}$ deposition in the microvasculature of the organ transplanted. C4d is a degradation product of the classic complement pathway. After an antigen-antibody complex fixes complement, a cascade of events follows with activation of several complement proteins. The complement protein $\mathrm{C} 4$ is split into $\mathrm{C} 4 \mathrm{a}$ and $\mathrm{C} 4 \mathrm{~b}$. C4b is then converted to $\mathrm{C} 4 \mathrm{~d}$ which can binds covalently to the endothelial and collagen basement membranes. Pathologic demonstration of C4d deposition has become an essential component of acute rejection [2,3]. C4d deposition in peritubular capillaries of renal allografts has been demonstrated to be a sensitive and diagnostic in-situ marker of acute humoral rejection that correlates strongly with the presence of circulating donor-specific antibodies. Biopsies with chronic allograft arteriopathy or glomerulopathy also have a high frequency of C4d deposition and donor-specific antibodies [4]

In contrast, the role of the complement system in bone marrow transplantation (BMT) is less well established. Bone marrow transplant, by virtue of the cellular components transplanted, resets not only the marrow itself, but ultimately resets the recipient's immune surveillance system. Unlike organ transplantation, the goal of BMT is to create tolerance that is life-long, and in best cases can develop without continued immunosuppression. Unfortunately, the entire recipient is prone to identification as a foreign antigen, causing multi organ damage termed graft-versus-host disease (GVHD). GVHD is a major cause of morbidity and mortality of allogeneic hematopoietic stem cell transplantation (HSCT). GVHD can occur in up to $50 \%$ of patients who receive sibling donor allogeneic hematopoietic cell and up to $70 \%$ from matched unrelated donor, 
despite early intensive prophylaxis with multiple immunosuppressive agents [5-8]. The diagnosis of GVHD is primarily made on the basis of clinical presentation with involvement of principal target organs, including the skin, liver, gastrointestinal tract, lung and hematopoietic system. Pathophysiologically, GVHD occurs when donor T-cells recognize the presence of foreign histocompatibility antigens in the host. Historically, CD4 T cells are thought crucial for maintaining the expansion of CD8 $\mathrm{T}$ cells primarily involved in GVHD pathogenesis $[1,9]$. The proliferation of activated T-cells leads to the production and secretion of a variety of cytokines which are responsible for the inflammatory effects and tissue damage associated with GVHD [10]. Histologically, this is seen by infiltration of the host tissue by lymphocytes, integration of lymphocytes and destruction of basic components of architecture of the host tissue, and finally complete obliteration of normal tissue. Grading of these events is often difficult pathologically, and subject to a large discrepancy [11].

More recently, the mechanisms involving GVHD pathogenesis have been re-evaluated. Recent advances in the treatment of GVHD have largely been in the arena where inclusion of other cellular subsets such as regulatory $\mathrm{T}$ cells, NK cells, and B cells have been targeted [12]. Despite inclusion of these new cellular components of the immune system, little has been evaluated in the innate immune systems such as pre-formed antibodies or complement fixation. Murine studies have demonstrated a strong correlation between the severity of GVHD in the skin and liver and the intensity of deposition of complement components (C5b-9) in these damaged organs $[13,14]$. Nishimura et al. have used anti-Mouse C5 antibodies to prevent and treat GVHD in mouse models and found that the use of these antibodies potentially ameliorate the symptoms of GVHD [14]. Furthermore, C3, C4 levels and circulating immune complexes were measured in 38 patients undergoing HLA-identical BMT and showed a strong association between the development of chronic GVHD and hypercomplementemia [15]. Recently, Zangh et al. reported three cases of chronic GVHD identified in colon biopsies in which dilated capillaries were all positively stained for $\mathrm{C} 4 \mathrm{~d}$, four months to two years following BMT [16].

To further study the role of the complement system in the pathogenesis of GVHD, the researchers at Oklahoma University Health Sciences Center (OUHSC) decided to test the correlation between the complement $\mathrm{C} 4 \mathrm{~d}$ endovascular deposition intensity and the severity of GVHD clinically.

\section{Material and Method}

\section{Study population}

This is a retrospective study that has been approved by the OUHSC institutional review board. All patients who developed clinical GVHD after hematopoietic stem cell transplantation and had biopsies at Oklahoma University Health Sciences Center between 2000 and 2008 were included in the study (cases with confirmed infectious etiologies were excluded). 73 biopsies from 61 patients were identified. Colon and skin biopsies were evaluated separately. For individuals with multiple biopsies of the same type, only the first biopsy was used in the study.

Eight skin biopsies had missing data, leaving 58 analyzable biopsies from 55 patients with GVHD: 40 skin and 18 colon. Three people had both colon and skin biopsy. Twelve colonic biopsies from nontransplant patients with relative normal colonic histology were used as control colon patients ( 6 biopsies from patients undergoing screening colonoscopies and 6 biopsies from patients having chronic diarrhea or from patients with quiescent ulcerative colitis or Crohn's). Colon biopsy controls from patient with chronic inflammatory bowel disease were chosen to evaluate the impact of history of chronic diarrhea on complement staining.

Clinical assessment for colon and skin GVHD was performed according to National Institutes of Health consensus criteria [11,17-19]. Clinical GVHD grading (grades I to IV) was used to determine the severity of GVHD and stratify patients accordingly. Cases were considered acute GVHD if the clinical manifestations occurred before day +100 of the stem cell transplant date, and chronic otherwise. Due to the limited number of cases in each category, the statistical analysis was performed by combining acute and chronic GVHD cases together.

Statistical analysis was performed using Fisher's exact test to compare between groups and controls. Alpha was set at 0.05 .

\section{C4d Immunohistochemistry}

Formalin-fixed paraffin-embedded tissues were cut at a thickness of $4 \mu \mathrm{m}$, mounted on SuperfrostÒPlus slides (Statlab Medical Products, Lewisville, TX) and subsequently deparaffinized, rehydrated, and washed in Tris Buffered Immunohistochemistry wash buffer + Tween ${ }^{\circ}$ 20 (Catalog \#935B, Cell Marque Rocklin, CA). Antigen retrieval (Dako Target Retrieval Solution, $\mathrm{pH}$ 9, DAKO, Carpinteria, CA) and endogenous peroxidase blocking (Catalog \#925B, Cell Marque, Rocklin, CA) were carried out following the manufacturers' instructions. The immunohistochemistry for $\mathrm{C} 4 \mathrm{~d}$ was performed with a rabbit polyclonal antibody (Catalog \#404A-18, Cell Marque, Rocklin, CA) using the HiDef detection HRP Mouse/Rabbit polymer system (Catalog \#954D, Cell Marque, Rocklin, CA). The immunohistochemistry was performed with adequate positive and negative controls.

\section{Histologic analysis}

Slides were examined utilizing the Olympus BX51 microscope. All routine Hematoxylin and eosin (H\&E) stained sections were reviewed to confirm the diagnosis. The C4d stained slides were examined and evaluated by an experienced pathologist (Z.Y). The grading was carried out using an adopting Banff 07 grading system for kidney allograft rejection [20]: Minimal staining was denoted as C4d positive capillaries affecting 1 to $10 \%$ of vessels, focal as affecting 10 to $50 \%$ of vessels and diffuse as affecting $>50 \%$ of vessels.

\section{Results}

C4d staining for endovascular deposition was performed on 58 GVHD biopsies (40 skin, 18 colon) and 12 controls (6 screening colonoscopy, 6 quiescent with history of inflammatory bowel) (Table 1).

All skin cases had histological features of GVHD (Figure 1A). Of 40 skin samples, 27 showed positive C4d staining (43\% minimal, 9\% focal, and $1 \%$ diffuse). Figure $1 \mathrm{~B}$ compared to 37 cases having diagnostic criteria for pathologic GVHD by H\&E (Table 2). Table 1 describes the density of staining. Staining did not appear to correlate well with steroid sensitivity: 20 (74\%) of 27 patients with positive C4d staining responded to steroid therapy while $12(92 \%)$ of 13 patients with negative C4d staining responded to steroid therapy, $\mathrm{p}=0.2634$ ) 
(Tables 3 and 4). Among the 30 grade I/II clinical skin GVHD, 19 (63\%) had complement deposition compared to $8(80 \%)$ out of the 10 cases with grade III/IV skin GVHD (Table 4). Of note, most skin cases had a relatively high background C4d staining; making accurate interpretation difficult.

Eighteen colon GVHD samples and 12 colon control samples were analyzed and compared (Table 1). Among clinical colon GVHD case, 8 had acute GVHD and 10 had chronic GVHD (Figure 2A). The proportion of patients with positive C4d staining was significantly greater in GVHD patients $(94 \%, 17$ of 18) as compared to control patients $(17 \%, 2$ of 12$)$ (p-value $<0.001)$ (Table 1). Among the 17 cases with positive C4d staining; 1 showed minimal, 4 showed focal and 12 showed diffuse C4d staining respectively (Table 1). All chronic GVHD and 7 out of 8 acute GVHD had C4d staining (Figure 2A). Only 8 (44\%) of the clinical colon GVHD patients have diagnostic criteria for pathologic GVHD by H\&E. The 10 remaining cases were all positive for C4d staining and 90\% (9/10) responded to steroid therapy (Figure 2B and Table 2). Among the 18 clinical colon GVHD patients, $44 \%$ (8/18) had a histologic diagnosis of GVHD (by H\&E), 61\% (11/18) had clinical grade III/IV, and 78\% (14/18) responded to steroid therapy (Figure 2C and 2D). It is worth noting that only $1(14 \%)$ of the 7 cases with grade I/II colon GVHD had pathologic confirmation of GVHD by H\&E (Table 4). The 10 clinical colon GVHD patients that did not have diagnostic criteria for pathologic GVHD by H\&E, were all positive for C4d staining and $90 \%(9 / 10)$ responded to steroid therapy (Figure 3A-3F).

As noted above, colon controls were split between benign screening colonoscopies and quiescent inflammatory bowel screening biopsies. The choice of these two populations was made to clarify the impact of treated inflammatory bowel, in an attempt to rule out the possibility that immunosuppressive therapy or previous inflammatory disease confounded finding of complement deposition. Among the 12 colon controls, $83 \%(10 / 12)$ were negative for C4d. Notably all 6/6 of screening colonoscopy samples were negative, and $4 / 6$ samples of quiescent inflammatory bowel were negative. The two cases of quiescent inflammatory bowel that were positive showed minimal staining (Table 1).

\begin{tabular}{|l|l|l|l|l|l|l|}
\hline \multicolumn{2}{|c}{} & \multicolumn{2}{l}{} \\
\cline { 3 - 7 } \multicolumn{2}{|c|}{ C4d IHC Results } & None & Minimal & Focal & Diffuse & Total \\
\hline Colon Cases* & Patient Cases (clinical GVHD) & 1 & 1 & 4 & 12 & 18 \\
\hline & Normal Controls & 10 & 2 & 0 & 0 & 12 \\
\hline Skin Cases & Patient Cases (clinical GVHD) & 13 & 17 & 9 & 1 & 40 \\
\hline "p-value<.0001, colon cases vs. controls & & & & \\
\hline
\end{tabular}

Table 1: C4d Immunohistochemistry (IHC) Results by Group.

\begin{tabular}{|c|c|c|c|c|c|c|c|c|}
\hline & & \multirow[b]{2}{*}{ Steroid Sensitivity } & \multicolumn{3}{|c|}{$\begin{array}{l}\text { Histopathology positive } \\
\text { for GVHD (by H\&E) }\end{array}$} & \multicolumn{3}{|c|}{$\begin{array}{l}\text { Histopathology negative } \\
\text { for GVHD ((by H\&E) }\end{array}$} \\
\hline & & & $\mathrm{C} 4 \mathrm{~d}+$ & C4d - & Total & C4d+ & C4d - & Total \\
\hline \multirow[t]{3}{*}{ Colon } & & Yes & 4 & 1 & 5 & 9 & 0 & 9 \\
\hline & & No & 3 & 0 & 3 & 1 & 0 & 1 \\
\hline & Total & & 7 & 1 & 8 & 10 & 0 & 10 \\
\hline \multirow[t]{3}{*}{ Skin } & & Yes & 18 & 11 & 29 & 2 & 1 & 3 \\
\hline & & No & 7 & 1 & 8 & 0 & 0 & 0 \\
\hline & Total & & 25 & 12 & 37 & 2 & 1 & 3 \\
\hline
\end{tabular}

Table 2: Colon biopsy cases: Comparison of histopathological diagnosis, steroid sensitivity, and C4d positivity.

\begin{tabular}{|c|c|c|c|c|c|c|c|}
\hline & & \multicolumn{6}{|c|}{ Clinical Grade } \\
\hline & & \multicolumn{3}{|c|}{ Grade I/II } & \multicolumn{3}{|c|}{ Grade III/IV } \\
\hline & & \multicolumn{3}{|c|}{ C4d IHC } & \multicolumn{3}{|c|}{ C4d IHC } \\
\hline & Steroid Sensitivity & C4d + & C4d- & Total & $\mathrm{C} 4 \mathrm{~d}+$ & C4d- & Total \\
\hline \multirow[t]{2}{*}{ Colon } & Yes & 6 & 0 & 6 & 7 & 1 & 8 \\
\hline & No & 1 & 0 & 1 & 3 & 0 & 3 \\
\hline
\end{tabular}


Citation: Cherry MA, Parekh H, Lerner M, Yu Z, Vesely SK, et al. (2015) The Role of Complement System in Graft versus Host Disease. J Blood Disord Transfus 6: 274. doi:10.4172/2155-9864.1000274

Page 4 of 7

\begin{tabular}{|l|l|l|l|l|l|l|l|l|}
\hline & Total & & 7 & 0 & 7 & 10 & 1 & 11 \\
\hline \multirow{3}{*}{ Skin } & & Yes & 17 & 11 & 28 & 3 & 1 & 4 \\
\cline { 2 - 10 } & & No & 2 & 0 & 2 & 5 & 1 & 6 \\
\cline { 2 - 9 } & Total & & 19 & 11 & 30 & 8 & 2 & 10 \\
\hline
\end{tabular}

Table 3: Colon and skin biopsy cases: Comparison of clinical grade, C4d IHC,and steroid sensitivity.

\begin{tabular}{|c|c|c|c|c|c|c|c|c|}
\hline & & & \multicolumn{6}{|c|}{ Clinical Grade } \\
\hline & & \multirow[b]{3}{*}{ H\&E status } & \multicolumn{3}{|l|}{ Grade I/II } & \multicolumn{3}{|c|}{ Grade III/IV } \\
\hline & & & \multirow{2}{*}{$\begin{array}{l}\text { C4d IHC } \\
\text { C4d + }\end{array}$} & \multicolumn{3}{|c|}{ C4d IHC } & \multirow[b]{2}{*}{ C4d- } & \multirow[b]{2}{*}{ Total $\mathbf{n}$} \\
\hline & & & & C4d- & $\begin{array}{l}\text { Total } \\
\text { n }\end{array}$ & $\mathrm{C} 4 \mathrm{~d}+$ & & \\
\hline \multirow[t]{3}{*}{ Colon } & & Positive & 1 & 0 & 1 & 6 & 1 & 7 \\
\hline & & Negative & 6 & 0 & 6 & 4 & 0 & 4 \\
\hline & Total $\mathrm{n}$ & & 7 & 0 & 7 & 10 & 1 & 11 \\
\hline \multirow[t]{3}{*}{ Skin } & & Positive & 17 & 10 & 27 & 8 & 2 & 10 \\
\hline & & Negative & 2 & 1 & 3 & 0 & 0 & 0 \\
\hline & Total $\mathrm{n}$ & & 19 & 11 & 30 & 8 & 2 & 10 \\
\hline
\end{tabular}

Table 4: Colon and Skin cases. Comparison of clinical grade, C4d IHC, and H\&E status.

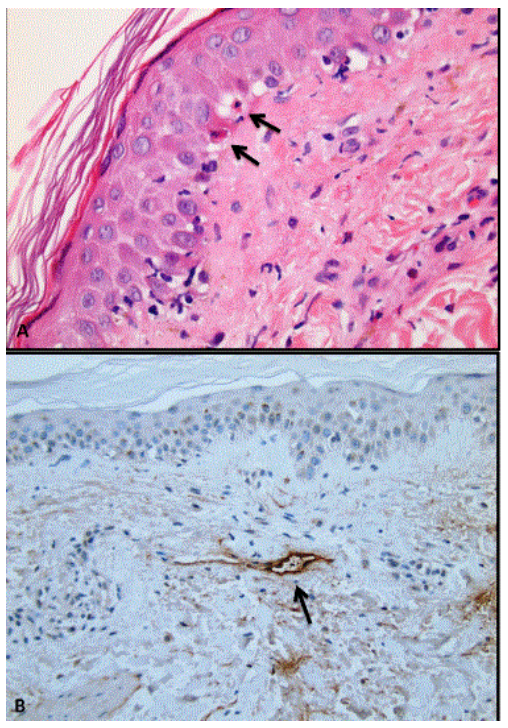

Figure 1: Skin biopsy from a patient with clinical features of GVHD. A: H\&E section shows classic histopathological features of GVHD with interface dermatitis and apoptotic keratinocytes (arrows) (40x original magnification); B: C4d immunostain on the same patient in 1A shows focal positive staining in small dermal vessels (arrow) (40x original magnification).

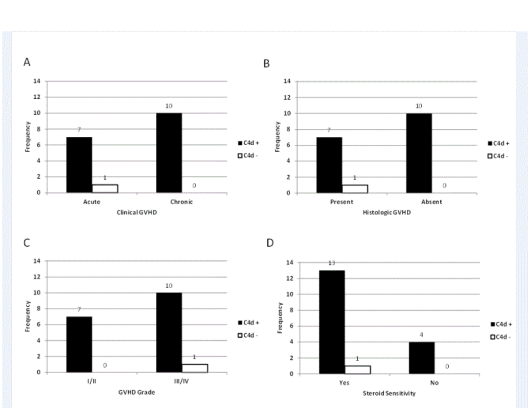

Figure 2: C4d staining in 18 colon GVHD biopsies. Black bars represent colon biopsies that are positive for C4dand white bars represent colon biopsies that are negative for C4dstaining. A: Of the 18 patients clinically diagnosed with colon GVHD, 17 were positive for C4d (94\%). B: Of the 8 patients with histologic GVHD present, 7 were positive for $\mathrm{C} 4 \mathrm{~d}(88 \%)$; of the 10 patients with histologic GVHD absent, all 10 (100\%) were positive for C4d. C: Among the 7 patients with GVHD grade I or II, all 7 were positive for C4d (100\%) and among the 11 patients with GVHD grade III or IV, 10 were positive for C4d (91\%). D: Among the 14 patients who were sensitive to steroids, 13 (93\%) were positive for C4d and among the 4 patients who were not sensitive to steroids, all 4 (100\%) were positive for C4d. 


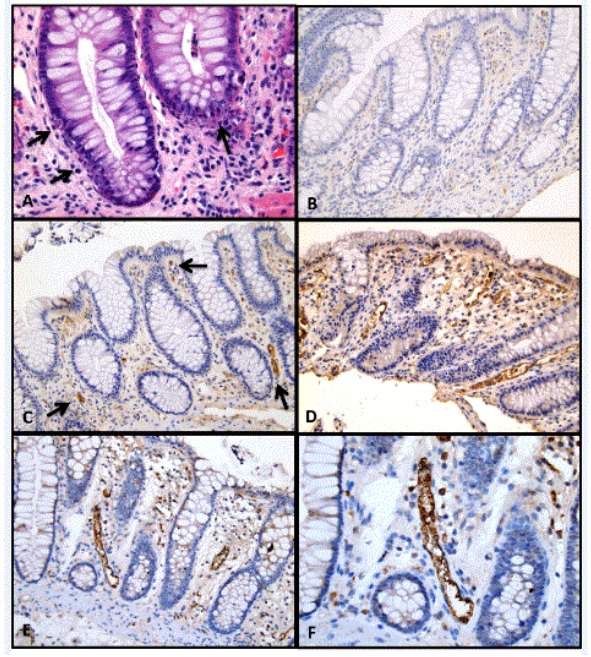

Figure 3: Colonic biopsy from patients with clinical features of GVHD. A: H\&E section shows classic histopathological features of GVHD with numerous apoptotic cryptic cells (arrows) (40x original magnification). B: one of the patients with histological features of GVHD shows negative C4d staining (20x original magnification); $\mathrm{C}$ and $\mathrm{D}$ : two separate patients with histological features of GVHD show focal (3C) and diffuse (3D) intramucosal vascular C4d staining (20x original magnification; arrows in 3C highlight the vascular structures). E: one patient without histological features of GVHD shows diffuse intramucosal vascular C4d staining (20x original magnification). F: higher magnification of $3 \mathrm{E}$ to highlight the accentuated C4d vascular staining (40x magnifications).

\section{Discussion}

Since the advent of bone marrow transplant as a curative option for hematologic malignancies, one of the major barriers to success has been graft versus host disease and morbidity/mortality of its treatment. GVHD and complications related to its treatment are responsible for $60 \%$ of transplant deaths [21]. Classically aimed at T-cell, and recently B-cell lymphocyte compartments of the immune system, highly immunosuppressive therapies such as steroids, monoclonal antibodies, and photopheresis remain unsuccessful in over $20-30 \%$ of cases $[22,23]$. Identification of other compartments of the immune system such as the complement system allow for the promise of identification of additional aspects of this disease as well as possible therapeutic venues. Long considered the key factor of graft rejection in solid tumor transplantation, complement is virtually unexplored in bone marrow transplantation. Considered a definitive marker of early rejection in solid tumor transplantation, identification of activity in bone marrow transplant is equally unknown. In contrast, GVHD is difficult to identify, and requires tissue destruction as a mainstay of consistent pathologic diagnosis. This requirement of destruction minimizes the possibility of early diagnosis and therapeutic impact when the opportunity for success is at its greatest.

Our study has identified that complement fixation is an early and consistent sign of GVHD as was demonstrated by its deposition in the majority of clinical colon GVHD (94\% of cases). We have identified that all clinical GVHD patients without diffuse staining responded to steroids compared to only $67 \%$ of patients with diffuse staining. This information can be used to estimate chances of response to steroids. Having diffuse staining may predict for lower chances of responding to steroids, and a lower threshold to switch therapy to second line agents should probably be opted at the earliest sign of resistance.

Like in solid tumor transplantation where C4d endovascular deposition has been demonstrated to be valuable in early graft rejection detection $[24,25]$, C4d in the bone marrow transplant host seems to be a key feature of early rejection or GVHD. It is possible that C4d is a more sensitive marker of clinical GVHD as it represents an earlier time point of GVHD before tissue destruction by $\mathrm{T}$ cell infiltration has occurred.

We have found that 7 out of 8 patients with grade I/II colon GVHD demonstrated positive C4d deposition compared to only 1 out of 7 detected by H\&E method (Figure 3). The standard H\&E method seems to detect more advanced GVHD grades and perhaps more clinically relevant cases. In our study, it is important to note that these changes seemed to be relatively isolated to GVHD patients, with no or minimal staining in other inflammatory conditions such as quiescent Crohn's and ulcerative colitis. This provides greater objectivity of early GVHD changes, allowing for more standard evaluation of therapeutic options. More importantly, $90 \%$ of colon cases that were negative by $\mathrm{H} \& \mathrm{E}$ but stained positive for C4d were responsive to steroid therapy, suggesting C4d staining may be a useful early indicator for GVHD. Although more studies are needed before its clinical application, C4d immunohistochemistry may assist in detecting early GVHD in the future and providing clinicians with an option to treat these patients empirically with steroids before searching for rarer conditions causing colitis (HHV6, Parasitic infections, etc...) and hence exposing patients to more laboratory testing and empiric antimicrobials and at the same time delaying steroids and immunosuppressive therapy.

This test (C4d immunohistochemistry) however lacks the sensitivity and specificity desired when it comes to skin GVHD. This is partly due to the paucity of the vasculature of the skin and the fact that there is higher background collagen staining for $\mathrm{C} 4 \mathrm{~d}$ which hampers optimal evaluation of endovascular immunostaining by the examining pathologist.

One of the limitations of this study is the fact that we chose our cases based on the clinical suspicion and not the pathologic confirmation by H\&E method. This decision was based on the fact that treatment for GVHD cases is often based on the clinical background regardless of the pathologic confirmation, and in many cases, clinicians would treat suspected GVHD cases with steroids or other immunosuppressants without even performing a biopsy. In addition, $\mathrm{H} \& \mathrm{E}$ method in diagnosing GVHD is not perfect as well as the concordance among pathologists.

Another limitation resides in combining both acute and chronic GVHD in the same analysis. In fact, the authors tried to separate skin and colon GVHD into acute and chronic but due to the low number of colon cases, it was difficult to draw any conclusion.

The third limitation is related to the retrospective nature of this study. To overcome this problem, we tried to include all cases (if infectious etiologies were ruled out) to minimize any selection bias.

Despite all these limitations, our study has clearly shown a potential role of the complement system in colon GVHD. The identification of the complement system as a key player in the pathogenesis of acute rejection in solid organ transplantation has opened the door to explore 
novel therapeutic applications targeting the complement system and potentially preventing acute organ rejection. The presence of complement deposition in early GVHD is intriguing, as Eculizumab, an antibody against C5 molecule, has been approved by the FDA for treatment of Paroxysmal Nocturnal Hemoglobinuria and atypical hemolytic uremia syndrome [26,27]. This has been tried successfully in kidney transplantation and it was found to decrease endothelial damage and prevent organ rejection $[28,29]$.

\section{Conclusion}

To the best of our knowledge, this is the largest preclinical study evaluating the role of complement system in the pathogenesis of GVHD. We have identified C4d deposition as a potentially valuable marker for early detection of colon GVHD. Further studies of complement in both of these venues, diagnostic and therapeutic, will open the opportunity to incorporate complement system alteration in the care of stem cell transplant patients. Further investigations into its potential may further clarify the impact of its diagnostic use and therapeutic implications.

\section{Authorship and Disclosures}

M.A.C designed the research and wrote the manuscript; H.P helped in collecting the data; M.L. processed the biopsy specimens and stained them for C4d deposition; Z.Y. read and interpreted all pathology specimens; S.K.V. analyzed the data; R.E. reviewed and edited the manuscript; G.S. reviewed and edited the manuscript; J.H. wrote and edited the manuscript.

We thank Dr. Carrie Yuen who reviewed and edited the manuscript.

This study was supported by Alexion pharmaceuticals that provided the reagent $(\mathrm{C} 4 \mathrm{~d})$ and compensation for the surgical technologist M.L. time.

All authors (M.A.C, H.P, M.L, Z.Y, S.K.V, R.E, G.S, and J.H) declare that they do not have any conflict of interest or anything to disclose concerning this study.

\section{References}

1. Lerner KG, Kao GF, Storb R, Buckner CD, Clift RA, et al. (1974) Histopathology of graft-vs.-host reaction (GvHR) in human recipients of marrow from HL-A-matched sibling donors. Transplant Proc 6: 367-371.

2. Kayler LK, Kiss L, Sharma V, Mohanka R, Zeevi A, et al. (2008) Acute renal allograft rejection: diagnostic significance of focal peritubular capillary C4d. Transplantation 85: 813-820.

3. Kieran N, Wang X, Perkins J, Davis C, Kendrick E, et al. (2009) Combination of peritubular c4d and transplant glomerulopathy predicts late renal allograft failure. J Am Soc Nephrol 20: 2260-2268.

4. Mauiyyedi S, Colvin RB (2002) Humoral rejection in kidney transplantation: new concepts in diagnosis and treatment. Curr Opin Nephrol Hypertens 11: 609-618.

5. Loiseau P, Busson M, Balere ML (2007) HLA Association with hematopoietic stem cell transplantation outcome: the number of mismatches at HLA-A, -B, -C, -DRB, or -DQB1 is strongly associated with overall survival. Biol Blood Marrow Transplant 13: 965-974.

6. Ratanatharathorn V, Nash RA, Przepiorka D, Devine SM, Klein JL, et al. (1998) Phase III study comparing methotrexate and tacrolimus (prograf, FK506) with methotrexate and cyclosporine for graft-versus-host disease prophylaxis after HLA-identical sibling bone marrow transplantation. Blood 92: 2303-2314.
7. Petersdorf EW, Longton GM, Anasetti C, Martin PJ, Mickelson EM, et al. (1995) The significance of HLA-DRB1 matching on clinical outcome after HLA-A, B, DR identical unrelated donor marrow transplantation. Blood 86: 1606-1613.

8. Flomenberg N, Baxter-Lowe LA, Confer D, Fernandez-Vina M, Filipovich A, et al. (2004) Impact of HLA class I and class II highresolution matching on outcomes of unrelated donor bone marrow transplantation: HLA-C mismatching is associated with a strong adverse effect on transplantation outcome. Blood 104: 1923-1930.

9. Janssen EM, Lemmens EE, Wolfe T, Christen U, von Herrath MG, et al. (2003) CD4+ T cells are required for secondary expansion and memory in CD8+ T lymphocytes. Nature 421: 852-856.

10. Ferrara JL, Deeg HJ (1991) Graft-versus-host disease. N Engl J Med 324: 667-674.

11. Shulman HM, Kleiner D, Lee SJ, Morton T, Pavletic SZ, et al. (2006) Histopathologic diagnosis of chronic graft-versus-host disease: National Institutes of Health Consensus Development Project on Criteria for Clinical Trials in Chronic Graft-versus-Host Disease: II. Pathology Working Group Report. Biol Blood Marrow Transplant. 12: 31-47.

12. Ball LM, Egeler RM; EBMT Paediatric Working Party (2008) Acute GvHD: pathogenesis and classification. Bone Marrow Transplant 41 Suppl 2: S58-64.

13. Niculescu F, Niculescu T, Nguyen P, Puliaev R, Papadimitriou JC, et al. (2005) Both apoptosis and complement membrane attack complex deposition are major features of murine acute graft-vs.-host disease. Exp Mol Pathol 79: 136-145.

14. Jun-ichi Nishimura DD, Benny J. Chen, Yuzuru Kanakura, Russell P. Rother, Nelson J. Chao (2007) Prevention of Graft-Versus-Host Disease in Mouse Model Using Anti-mouse C5 Antibody. [Abstract]. blood 118: 953a.

15. Varga L, Poros A, Puskás E, Pánya A, Kramer J, et al. (1995) Clinical significance of longitudinal complement measurements in recipients of bone marrow transplant. Bone Marrow Transplant 15: 509-514.

16. Zhang PL, Wilkerson ML, Schworer CM (2008) C4d staining is a valuable marker in identifying chronic GVHD in colonic biopsies following BMT. Bone Marrow Transplant 42: 209-211.

17. Przepiorka D, Weisdorf D, Martin P, Klingemann HG, Beatty P, et al. (1995) 1994 Consensus Conference on Acute GVHD Grading. Bone Marrow Transplant 15: 825-828.

18. Sale GE, Lerner KG, Barker EA, Shulman HM, Thomas ED (1977) The skin biopsy in the diagnosis of acute graft-versus-host disease in man. Am J Pathol 89: 621-636.

19. Filipovich AH, Weisdorf D, Pavletic S, Socie G, Wingard JR, et al. (2005) National Institutes of Health consensus development project on criteria for clinical trials in chronic graft-versus-host disease: I. Diagnosis and staging working group report. Biol Blood Marrow Transplant 11: 945-956.

20. Solez K, Colvin RB, Racusen LC, Haas M, Sis B, et al. (2008) Banff 07 classification of renal allograft pathology: updates and future directions. Am J Transplant 8: 753-760.

21. Pasquini MC (2008) Impact of graft-versus-host disease on survival. Best Pract Res Clin Haematol 21: 193-204.

22. Greinix HT, Volc-Platzer B, Kalhs P, Fischer G, Rosenmayr A, et al. (2000) Extracorporeal photochemotherapy in the treatment of severe steroid-refractory acute graft-versus-host disease: a pilot study. Blood 96: 2426-2431.

23. Zaja F, Bacigalupo A, Patriarca F, Stanzani M, Van Lint MT, et al. (2007) Treatment of refractory chronic GVHD with rituximab: a GITMO study. Bone Marrow Transplant 40: 273-277.

24. Archdeacon P, Chan M, Neuland C, Velidedeoglu E, Meyer J, et al. (2011) Summary of FDA antibody-mediated rejection workshop. Am J Transplant 11: 896-906.

25. Feucht HE, Felber E, Gokel MJ, Hillebrand G, Nattermann U, et al. (1991) Vascular deposition of complement-split products in kidney allografts with cell-mediated rejection. Clin Exp Immunol 86: 464-470. 
Citation: Cherry MA, Parekh H, Lerner M, Yu Z, Vesely SK, et al. (2015) The Role of Complement System in Graft versus Host Disease. J Blood Disord Transfus 6: 274. doi:10.4172/2155-9864.1000274

Page 7 of 7

26. Brodsky RA, Young NS, Antonioli E, Risitano AM, Schrezenmeier H, et al. (2008) Multicenter phase 3 study of the complement inhibitor eculizumab for the treatment of patients with paroxysmal nocturnal hemoglobinuria. Blood 111: 1840-1847.

27. Muus PLC, Douglas K (2010) Safety and efficacy of eculizumab in aHUS patients on chronic plasma therapy: interim analysis of a phaseII trial (FPO1274) [abstract]. J Am Soc Nephrol 21: (402A.).
28. Chatelet V, Frémeaux-Bacchi V, Lobbedez T, Ficheux M, Hurault de Ligny B (2009) Safety and long-term efficacy of eculizumab in a renal transplant patient with recurrent atypical hemolytic-uremic syndrome. Am J Transplant 9: 2644-2645.

29. Cornell LB, Stegall JM, (2009) Prevention of Endothelial. Activation with C5 Inhibition in Positive-Crossmatch Kidney Transplants (+XMKTx). American transplant Congress. 